\title{
A CHARACTERISATION OF ABSOLUTELY CONTINUOUS MEASURES ON TOPOLOGICAL SEMIGROUPS
}

\author{
HENERI A. M. DZINOTYIWEYI
}

(Communicated by Roe Goodman)

\begin{abstract}
Let $S$ be a completely regular topological semigroup and $\mu$ a bounded regular Borel measure on $S$. For a very large class of noncompact semigroups $S$, we show that the map $x \rightarrow \mu^{*} \bar{x}$ of $S$ into the space of bounded regular Borel measures on $S$ is norm-continuous if and only if $\mu_{0} f$ is a left uniformly continuous function on $S$, for all bounded continuous functions $f$ on $S$. Here the function $\mu_{0} f$ is given by
\end{abstract}

$$
\mu_{0} f(x):=\int f(y x) d \mu(y) \text { on } S .
$$

\section{INTRODUCTION}

Let $S$ be a completely regular topological semigroup, $C(S)$ the space of all bounded continuous functions on $S$, and $M(S)$ the algebra of all bounded regular Borel measures on $S$ with the usual total variation norm and convolution given by

$$
\nu^{*} \mu(f):=\iint f(x y) d \nu(x) d \mu(y)
$$

for all $\nu$ and $\mu$ in $M(S)$ and $f$ in $C(S)$.

For a measure $\mu$ in $M(S)$ and a function $f$ in $C(S)$, we have the function $\mu_{0} f$ in $C(S)$ given by

$$
\mu_{0} f(x):=\int f(y x) d \mu(y) \quad(x \in S) .
$$

The space of all bounded left uniformly continuous functions on $S$ is $\operatorname{LUC}(S):=\left\{f \in C(S)\right.$ : the map $x \rightarrow{ }_{x} f$ of $S$ into $C(S)$ is norm continuous $\}$ where

$$
{ }_{x} f(y):=f(x y) \quad(x, y \in S) .
$$

For a noncompact locally compact topological group $G$, a characterisation of $L^{1}(G)$-namely, that a measure $\mu$ in $M(G)$ is absolutely continuous to the Haar measure of $G$ if and only if the function $\mu_{0} f$ is left uniformly continuous for all $f$ in $C(G)$-is proved in [4]. The purpose of this paper is to extend

Received by the editors February 20, 1992 and, in revised form, November 4, 1992. 1991 Mathematics Subject Classification. Primary 43A20, 43A10; Secondary 22A20. 
that result to topological semigroups. For the latter, analogues of $L^{1}(G)$ are the so-called spaces of absolutely continuous measures on $S$, such as

$M_{n}^{r}(S):=\left\{\mu \in M(S)\right.$ : the map $x \rightarrow \mu^{*} \bar{x}$ of $S$ into $M(S)$ is norm continuous $\}$ and

$M_{a}(S):=\left\{\mu \in M(S):\right.$ the maps $x \rightarrow \bar{x}^{*}|\mu|(K)$ and $x \rightarrow|\mu|^{*} \bar{x}(K)$ of $S$ into $\mathbb{R}$ are continuous, for all compact $K \subset S$ \}.

For an extensive study of such spaces, we refer the reader to [2]. Other references include [1] and [6]. In particular it is well known that $M_{n}^{r}(S)=$ $M_{a}(S)$ for many semigroups that include locally compact topological groups as a "very" special case (cf. [2] or [6]).

Before stating our main results, we clarify the following notation: For $A_{1}, \ldots, A_{n}$ subsets of $S$ and $x$ an element of $S$, we define

$$
\begin{aligned}
A_{1}^{-1} A_{2} & :=\left\{y \in S: a y \in A_{2} \text { for some } a \in A_{1}\right\}, \\
x^{-1} A_{1} & :=\{x\}^{-1} A_{1} \text { and } A_{1}^{-1} x:=A_{1}^{-1}\{x\} .
\end{aligned}
$$

We similarly define the right-handed cases of the preceding notation. We also define

$$
\begin{aligned}
A_{1} \otimes_{l} A_{2} & :=\left\{A_{1} A_{2}, A_{1}^{-1} A_{2}\right\}, \\
A_{1} \otimes_{l} A_{2} \otimes_{l} A_{3} & :=\bigcup\left\{A_{1} \otimes_{l} B: B \in A_{2} \otimes_{l} A_{3}\right\},
\end{aligned}
$$

and hence, inductively, similarly define $A_{1} \otimes_{l} \cdots \otimes_{l} A_{n}$.

A subset $E$ of $S$ is said to be left relatively neocompact if $E$ is contained in a finite union of sets of the type of elements of $A_{1} \otimes_{l} \cdots \otimes_{l} A_{n}$ where $A_{1}, \ldots, A_{n}$ are some compact subsets of $S$. In particular, we note that if $C^{-1} D$ is compact for all compact subsets $C$ and $D$ of $S$, then $E$ is left relatively neocompact if and only if $E$ is relatively compact.

One extensively studied class of semigroups is that of stips (see, e.g., $[2,6]$ ). A locally compact topological semigroup $S$ with an identity element 1 is called a stip if for every neighbourhood $U$ of 1 we have that

$$
1 \in \operatorname{int}\left(U^{-1} u \cap v U^{-1}\right) \text { for some } u, v \text { in } U
$$

and

$$
x \in \operatorname{int}\left(U^{-1}(U x) \cap(x U) U^{-1}\right) \text { for all } x \text { in } S .
$$

We have $M_{a}(S)=M_{n}^{r}(S)$ for a stip $S$. Also a locally compact topological semigroup $S$, with an identity element and such that the support of $M_{a}(S)$ coincides with $S$, is a stip (see [2] or [6]).

Finally, before stating our results, we recall that $S$ is said to be right cancellative if $x y=z y$ implies that $x=z$ for all $x, y$, and $z$ in $S$.

\section{THE MAIN RESULTS}

1. Theorem. Let $S$ be a right cancellative, noncompact, $\sigma$-compact, and locally compact topological semigroup such that $C^{-1} D$ is compact for all compact subsets $C$ and $D$ of $S$, and let $\mu$ be a measure in $M(S)$. Then the following items are equivalent:

(i) The map $x \rightarrow \mu^{*} \bar{x}$ of $S$ into $M(S)$ is norm continuous. 
(ii) The function $\mu_{0} f$ is left uniformly continuous for all functions $f$ in $C(S)$.

For stips and for other semigroups supporting absolutely continuous measures, we have the following more general version of the preceding result.

2. Theorem. Let $S$ be a right cancellative completely regular topological semigroup that is a $K$-space and not left relatively neocompact, and let $\mu$ be a measure in $M(S)$. If either $M_{a}(S)$ is nonzero or $S$ is a stip, the following items are equivalent:

(i) The map $x \rightarrow \mu^{*} \bar{x}$ of $S$ into $M(S)$ is norm continuous.

(ii) The function $\mu_{0} f$ is left uniformly continuous for all $f$ in $C(S)$.

We then deduce the following generalization of a result of [5] (see also [3] for a related result.

3. Corollary. Let $S$ be a right cancellative stip such that $C^{-1} D$ is compact for all compact subsets $C$ and $D$ of $S$. Then

$$
C(S)=\operatorname{LUC}(S) \text { if and only if } S \text { is either compact or discrete. }
$$

\section{KEY LEMMAS}

1. Lemma. Let $S$ be a $\sigma$-compact, noncompact, and locally compact topological semigroup such that $A^{-1} B$ is compact for all compact subsets $A$ and $B$. If $K$ and $C$ are compact subsets, we can find a sequence $\left\{a_{n}\right\}$ of points of $S$ and a sequence $\left\{H_{n}\right\}$ of subsets of $S$ such that

(a) $H_{n}$ is a compact neighbourhood of $K C a_{n}$,

(b) the sets $(K C)^{-1} H_{n}$ are pairwise disjoint,

(c) each point of $S$ has a neighborhood that intersects only finitely many of the sets $H_{n}$,

for all $n$ in $\mathbb{N}$.

Proof. Since $S$ is $\sigma$-compact, there exists an increasing sequence of open and relatively compact sets, $U_{n}$, whose union is $S$. We may assume without loss of generality that $K$ and $C$ contain the identity element by adding one if necessary.

By the inductive hypothesis, suppose $\left\{a_{1}, \ldots, a_{m}\right\},\left\{H_{1}, \ldots, H_{m}\right\}$, and an increasing subsequence $\left\{U_{k_{1}}, \ldots, U_{k_{m}}\right\}$ have been chosen, for some $m \in \mathbb{N}$, such that (a) and (b) hold for all $n \leq m$ and

$$
\begin{array}{ll}
H_{i} \subseteq U_{k_{j}} & \text { for } i \leq j \leq m, \\
H_{i} \cap U_{k_{j}}=\varnothing & \text { for } j<i \leq m .
\end{array}
$$

As finite unions of sets of the form $A^{-1} B$, where $A$ and $B$ are compact, are also compact and as $S$ is not compact, we can choose $a_{m+1}$ a point in $S$ and $H_{m+1}$ a compact neighbourhood of $K C a_{m+1}$ such that (b) holds for $n \leq m+1$ and $H_{m+1} \cap \bar{U}_{k_{j}}=\varnothing$ if $j \leq m$.

Next we choose from the $U_{n}$ 's a set $U_{k_{m+1}}$ such that

$$
H_{m+1} \cup U_{k_{j}} \subseteq U_{k_{m+1}} \text { if } j \leq m .
$$


We thus have (1) holding for $i, j \leq m+1$, which completes our proof for the inductive step. Repeating the argument countably many times we obtain the required sequences satisfying (a) and (b) of our lemma.

To show that (c) also holds, fix any compact neighbourhood $X$ and note that $X \subseteq U_{k_{t}}$ for some $t$ in $\mathbb{N}$. Recalling (1) it follows that $X \cap H_{i}=\varnothing$ for all $i>t$. This completes our proof.

2. Lemma. Let $S$ be a completely regular topological semigroup that is not relatively neocompact and $K$ and $C$ compact subsets of $S$. If either $M_{a}(S)$ is nonzero or $S$ is a stip, there is a sequence $\left\{a_{n}\right\}$ of points of $S$ and a sequence $\left\{H_{n}\right\}$ of subsets of $S$ such that

(a) $H_{n}$ is a neighbourhood of $K C a_{n}$,

(b) the sets $(K C)^{-1} H_{n}$ are pairwise disjoint,

(c) each point of $S$ has a neighbourhood that intersects only finitely many of the sets $H_{n}$,

for all $n$ in $\mathbb{N}$

Proof. If $M_{a}(S)$ is nonzero, let $D$ be a compact subset of $S$ such that $|\eta|(D)>$ 0 for some measure $\eta$ in $M_{a}(S)$; and, if $S$ is a stip, let $D$ be a compact neighbourhood of the identity element. Then in both cases we have that

(*) $\quad D^{-1}(D F)$ is a neighbourhood of $F$ for every subset $F$ of $S$.

For a proof of $(*)$, see, e.g., [2, Lemma 2.1.9], for the case where $M_{a}(S)$ is nonzero, and, for the case of a stip, it is an immediate consequence of the definition.

We may assume without loss of generality that $K$ and $C$ contain an identity element

Given $a_{n}$ we define

$$
H_{n}:=D^{-1}\left(D K C a_{n}\right), \quad P_{n}:=D^{-1}\left(D H_{n}\right)
$$

and note that $H_{n}$ is a neighbourhood of $K C a_{n}$ by $(*)$. By the inductive hypothesis, suppose we have $m \in \mathbb{N}$ such that

$$
(K C)^{-1} P_{i} \cap(K C)^{-1} P_{j}=\varnothing \text { for distinct } i, j \leq m .
$$

Since $S$ is not relatively neocompact, we can choose $a_{m+1}$ in $S$ such that

$$
a_{m+1} \notin(D K C)^{-1}\left(D\left(D^{-1}\left(D K C\left((K C)^{-1} P_{i}\right)\right)\right)\right)
$$

which is equivalent to

$$
(K C)^{-1} P_{m+1} \cap(K C)^{-1} P_{i}=\varnothing
$$

for $i \leq m$. Repeating the argument countably many times we obtain the sequence $\left\{a_{n}\right\}$ such that the sets $(K C)^{-1} P_{n}$ are pairwise disjoint. Since $H_{n} \subseteq P_{n}$, we thus have (a) and (b) of our lemma.

To prove (c) let $x$ be any point of $S$ and recall that $D^{-1}(D x)$ is a neighbourhood of $x$ by $(*)$. If $D^{-1}(D x) \cap H_{k} \neq \varnothing$ for some $k$ in $\mathbb{N}$ then $x \in P_{k}$. Since the $P_{n}$ 's are pairwise disjoint, we then get

$$
D^{-1}(D x) \cap H_{n}=\varnothing \text { for } n \neq k .
$$

Hence (c) follows and our proof is complete. 
ThE PROOF OF TheOREMS 1 AND 2

We give a common proof for our two theorems. Accordingly let $S$ be a topological semigroup satisfying the hypothesis of either Theorem 1 or 2 .

That (i) implies (ii) is well known and follows from the fact that

$$
\begin{aligned}
\left\|_{X}\left(\mu_{0} f\right)-{ }_{y}\left(\mu_{0} f\right)\right\|: & =\sup \left\{\left|\mu_{0} f(x s)-\mu_{0} f(y s)\right|: s \in S\right\} \\
& =\sup \left\{\left|\mu^{*} \overline{x s}(f)-\mu^{*} \overline{y s}(f)\right|: s \in S\right\} \\
& \leq \sup \left\{\left\|\mu^{*} \overline{x s}-\mu^{*} \overline{y s}\right\|\|f\|_{S}: s \in S\right\} \\
& \leq\left\|\mu^{*} \bar{x}-\mu^{*} \bar{y}\right\|\|f\|_{S}
\end{aligned}
$$

for all $x$ and $y$ in $S$ and $f$ in $C(S)$.

We now prove that (ii) implies (i). Since $S$ is a $k$-space, it is evident that the norm continuity of the map $x \rightarrow \mu^{*} \bar{x}$ is equivalent to the norm compactness of the sets $\left\{\mu^{*} \bar{x}: x \in K\right\}$ for all compact subsets $K$ of $S$ (see, e.g., [2, Lemma 2.2.2]). So, if on the contrary the map $x \rightarrow \mu^{*} \bar{x}$ is not continuous, we can find a compact subset $C$ of $S$ whose image is not compact. We can pick a sequence $\left\{x_{n}\right\}$ in $C$ for which the image has no cluster point. On the other hand there exists a cluster point $x$ of $\left\{x_{n}\right\}$ in $C$. By omitting finitely many points, we may assume there exists an $\varepsilon>0$ such that

$$
\left\|\mu^{*} \bar{x}_{n}-\mu^{*} \bar{x}\right\|>5 \varepsilon \text { for all } n \text { in } \mathbb{N} \text {. }
$$

By the inner regularity of $\mu$, we can choose a measure $\nu$ in $M(S)$ with compact support $K$ such that $\|\nu-\mu\|<\varepsilon$. Consequently

$$
\left\|\nu^{*} \bar{x}_{n}-\nu^{*} \bar{x}\right\|>3 \varepsilon \text { for all } n \text { in } \mathbb{N} \text {. }
$$

Let the sequence $\left\{a_{n}\right\}$ and sets $H_{n}$ be chosen, with respect to $C$ and $K$, as in Lemma 1 or 2 for the corresponding semigroup $S$.

Since $S$ is right cancellative, we have

$$
\left\|\nu^{*} \overline{x_{n} a_{n}}-\nu^{*} \overline{x a_{n}}\right\|=\left\|\nu^{*} \bar{x}_{n}-\nu^{*} \bar{x}\right\|>3 \varepsilon \text { for all } n \text { in } \mathbb{N} \text {. }
$$

So we can find functions $f_{n}$ such that $\left\|f_{n}\right\|=1$ and

$$
\left|\left(\nu^{*} \overline{x_{n} a_{n}}-\nu^{*} \overline{x a_{n}}\right)\left(f_{n}\right)\right|>3 \varepsilon \text { for all } n \text { in } \mathbb{N} .
$$

There are positive continuous functions $\varphi_{n}$ of norm 1 , with value 1 on $K C a_{n}$ and vanishing outside $H_{n}$. Taking $h_{n}:=f_{n} \varphi_{n}$ we then get

$$
\left|\left(\nu^{*} \overline{x_{n} a_{n}}-\nu^{*} \overline{x a_{n}}\right)\left(h_{n}\right)\right|>3 \varepsilon \text { for all } n \text { in } \mathbb{N} .
$$

Without loss of generality, we may assume that both $K$ and $C$ contain the identity element of $S$, by adding one if necessary. Then the (b)'s in our lemmas imply that the sets $H_{n}$ are pairwise disjoint. Consider the function $h:=\sum_{n=1}^{\infty} h_{n}$, and note that $\|h\|=1$ since the $h_{n}$ 's are supported on disjoint sets $H_{n} ; h$ is continuous, by (c) in our lemmas.

The (ii)'s in our theorems show that $\mu_{0} h$ is left uniformly continuous. So we can find a neighbourhood $V$ of $x$ such that

$$
\left\|_{y}\left(\mu_{0} h\right)-{ }_{x}\left(\mu_{0} h\right)\right\|<\varepsilon \text { for all } y \in V .
$$


Recalling our choice of $\nu$ with respect to $\mu$ we get

$$
\left\|_{y}\left(\nu_{0} h\right)-{ }_{x}\left(\nu_{0} h\right)\right\|<3 \varepsilon \text { for all } y \text { in } V \text {. }
$$

Equivalently

$$
\left|\nu_{0} h(y s)-\nu_{0} h(x s)\right|<3 \varepsilon \quad(y \text { in } V \text { and } s \text { in } S) .
$$

Suppose

$$
\int h_{m}(t y s) d \nu(t)=\nu_{0} h_{m}(y s) \neq 0 \text { for some } y \in V \cap C \text { and } s \text { in } S .
$$

Then

$$
\text { tys } \in H_{m} \text { for some } t \text { in } K \text {, }
$$

so $s \in(K C)^{-1} H_{m}$. By the (b)'s in our lemmas, we then get

$$
\nu_{0} h_{m}(y s) \neq 0 \text { implies } \nu_{0} h_{n}(u s)=0 \text { for } n \neq m
$$

and $(u, y \in V \cap C$ and $s \in S)$. Consequently

$$
\left|\nu_{0} h_{n}(y s)-\nu_{0} h_{n}(x s)\right|<3 \varepsilon \quad(y \in V \cap C \text { and } s \in S) .
$$

Since $x$ is a cluster point of $\left\{x_{n}\right\}$ in $C$, there exist infinitely many $x_{n}$ 's in $V \cap C$. For such $n$, taking $s:=a_{n}$ we get

$$
\left|\left(\nu^{*} \overline{x_{n} a_{n}}-\nu^{*} \overline{x a_{n}}\right)\left(h_{n}\right)\right|=\left|\nu_{0} h_{n}\left(x_{n} a_{n}\right)-\nu_{0} h_{n}\left(x a_{n}\right)\right|<3 \varepsilon .
$$

Now $(\beta)$ contradicts $(\alpha)$. By this contradiction, we conclude that (ii) implies (i) (in our theorems).

\section{The PROOF OF COROLlaRY 3}

If $C(S)=\operatorname{LUC}(S)$, then $\mu_{0} f \in \operatorname{LUC}(S)$ for all $f$ in $C(S)$ and $\mu$ in $M(S)$. So, if $S$ is noncompact then $\mu \in M_{n}^{r}(S)$ for all $\mu \in M(S)$, by Theorem 2. In particular, $\bar{x} \in M_{n}^{r}(S)$ for all $x \in S$, so

$$
\begin{aligned}
O(y) & :=\left\{s \in S:\left\|\bar{x}^{*} \bar{s}-\bar{x}^{*} \bar{y}\right\|<1\right\} \\
& =\{s \in S:\|\overline{x s}-\overline{x y}\|<1\}=\{s \in S: x s=x y\}=x^{-1}(x y)
\end{aligned}
$$

is an open neighbourhood of $y$ for all $x, y$ in $S$. Now taking $x=1$, the identity of $S$, we get that $x^{-1}(x y)=\{y\}$ is open for all $y$ in $S$, so $S$ is discrete.

The remainder of our proof is well known and follows from the definitions.

\section{REMARKS}

1. If we drop the condition that $S$ be right cancellative in Theorem 1 , then the result may not hold. For a counterexample, we consider

$$
S:=\mathbb{N} \cup\left\{m+2^{-n}: m, n \in \mathbb{N}\right\} \quad \text { where } x y:=\max (x, y),
$$

for all $x, y \in S$, and the topology is the restriction of the line topology. Then the reader can easily verify that $C(S)=\mathrm{LUC}(S)$, so $\mu_{0} f$ is in $\mathrm{LUC}(S)$ for all $\mu$ in $M(S)$ and $f$ in $C(S)$. But $M_{n}^{r}(S)$ is the zero space $\{0\}$.

2. We say a topological semigroup $S$ is left semidiscrete if $x^{-1}(x y)$ is a neighbourhood of $y$ for all $x, y$ in $S$. In particular, if $S$ has an identity element or is left cancellative, then $S$ is left semidiscrete if and only if $S$ is discrete. 
Now let $S$ be a locally compact topological semigroup with $C^{-1} D$ compact for all compact subsets $C$ and $D$ and such that $M_{a}(S)$ is nonzero. Then the reader can easily extract from our proof of Corollary 3 that $S$ is left semidiscrete if $C(S)=\mathrm{LUC}(S)$. It remains an open problem whether or not $C(S)=\operatorname{LUC}(S)$ when $S$ is left semidiscrete.

3. For topological semigroups $S$ with either $M_{a}(S)$ nonzero or $S$ being a stip, we believe the cancellative condition may be dropped in our results and raise the following problems:

3.1. Conjecture. Theorem 2 holds without the right cancellative condition on $S$.

3.2. Conjecture. Corollary 3 holds without the right cancellative condition on $S$.

\section{ACKNOWLEDGMENT}

This research was partially done at T. H. Darmstadt, Germany, and we wish to thank Professor Dr. K. Keimel for inspiring discussions. Thanks are also due to the referee for simplifying parts of the original proof.

\section{REFERENCES}

1. A. C. Baker and J. W. Baker, Algebras of Measures on a locally compact topological semigroup II, J. London Math. Soc. (2) 2 (1970), 651-659.

2. H. A. M. Dzinotyiweyi, The analogue of the group algebra for topological semigroups, Pitman Advanced Publishing Program, Boston, London, and Melbourne, 1984.

3. __ Uniformly continuous functions on some topological semigroups, Quart. J. Math. Oxford Ser. 237 (1986), 11-16.

4. H. A. M. Dzinotyiweyi and A. C. M. van Rooij, $A$ characterization of the group algebra of a noncompact locally compact topological group, Quart. J. Math. Oxford Ser. 241 (1990), 15-20.

5. G. L. Itzkowitz, Uniform structure in topological groups, Proc. Amer. Math. Soc. 57 (1976), 363-366.

6. G. L. G. Sleijpen, Locally compact semigroups and continuous translations of measures, Proc. London Math. Soc. (2) 37 (1978), 75-9?.

Department of Mathematics, University of Zimbabwe, P. O. Box MP 167, Mount PleasANT, HARARE, ZimbabWe 